\title{
Electron Holography Studies of Amorphous Materials
}

\author{
Xidong Chen* and Dean Miller** \\ * Materials Science Division, Argonne National Lab, Argonne, IL 60439 \& Cedarville University, \\ Cedarville, $\mathrm{OH} 45314$ \\ ** Materials Science Division, Argonne National Lab, Argonne, IL 60439
}

Electron holography uses electron wave interference patterns to record both the amplitude and the phase information of an electron wave that passes through a sample. The phase information has been considered particularly interesting, as it can be used to determine the electrostatic potentials in the specimen. Therefore electron holography has been applied to measure the two-dimensional electrostatic potentials in semiconductor devices. [1] Amorphous materials, due to their lack of longrange ordering, however seem difficult for electron holography studies. In this paper, we explore potential applications of off-axis electron holography in studying amorphous materials.

The first part of this study will focus on using electron holography to conduct fluctuation microscopy studies. Fluctuation microscopy is a relatively new technique that probes medium-range ordering in amorphous materials [2]. There have already been many fluctuation microscopy studies of a variety of amorphous materials in recent years [3]. A current development of fluctuation microscopy is to develop a capability of varying the resolution. This capability would make it possible to study medium-range ordering in amorphous materials at different length scales. An approach is to use scanning transmission electron microcopy (STEM) mode. In this approach, the beam size is varied and then scanned through a sample. In this paper, we are suggesting an alternative approach that uses electron holography. Since electron holography can directly yield information on the wave amplitude and the phase, fluctuation microscopy may be directly conducted with the wave amplitude and the phase. First, a Fourier transform of the wave image is obtained. Numerical apertures of different sizes then can be easily applied to mask the Fourier transformed image at a particular transfer momentum $\mathrm{k}$ value. An inverse Fourier transform yields an image that corresponds to a dark filed image taken at the same $\mathrm{k}$ under a particular resolution. The variance then can be measured from this image. An advantage of this approach is that both the kalue and the resolution can be easily varied numerically. In this study, we tested this approach on amorphous diamond like-carbon films. Details of our preliminary results will be presented. Limitations of the current approach and further instrumentation requirements will be discussed.

The second part of this study will focus on measuring the electrostatic potentials in amorphous materials. In general, due to lack of long-range ordering, the electrostatic potentials in amorphous materials is stochastic and follows a probability distribution. This probability distribution was assumed to take certain functional forms in early works [4]. It has been however recently shown that medium-range ordering is directly related to electrical and optical property changes in amorphous diamond like carbon films [5]. It would be desirable to be able to experimentally measure the probability distribution of the electrostatic potentials in those films and see if mediumrange ordering affects the probability distribution. This could shed light how medium-range ordering is related to electrical and optical properties in those films. In our approach, a Fourier transform of a hologram is conducted. Then sidebands are isolated and copied into a new image. Both side bands are utilized to paste a full k-range image. Then a Fourier transform of this image yields a complex wave image with information on both the amplitude and the phase. Then the phase is directly measured from this wave image. Finally a two-dimensional electrostatic potential map is acquired 
from the phase image. The histogram of this potential map gives the probability distribution of the potential. Details of our results with this approach will be presented. This work was funded by Department of Energy-Basic Energy Science through the Electron Microscopy Center at Argonne National Laboratory.

\section{References:}

[1] A.C. Twichett et al. Phys. Rev. Lett. 88 (2002) 238302; W. D. Rau et al. Phys. Rev. Lett. 82 (1999) 2614; M.A. Gribelyuk et al. Phys. Rev. Lett. 89 (2002) 025502.

[2] J. M. Gibson and M.M. J. Treacy, Phys. Rev. Lett., 78 (1997)1074.

[3] P. M. Voyles, J. E. Gerbi, M. M. J. Treacy, J. M. Gibson, and J. R. Abelson, Phys. Rev. Lett. 86, (2001) 5514; Xidong Chen, John Sullivan, Tom Friedmann, and J. Murray Gibson, Appl. Phys. Lett., 84 (2004) 2823; Xidong Chen, John Sullivan, Charles Barbour, Craig Johnson, Guangwen Zhou, Judith Yang, Symposium G 1.4, Mat. Res. Soc. Symp. Proc. 738, 2002.

[4] P. W. Anderson, Phys. Rev. 109 (1958) 1492.

[5] Xidong Chen, John Sullivan, Tom Friedmann, and J. M. Gibson, Appl. Phys. Lett. 84, (2004) 2823; Xidong. Chen, J. M. Gibson, J. Sullivan and T. Friedmann, Symposium W, Materials Research Society Spring meeting, April 16 -20, 2001.

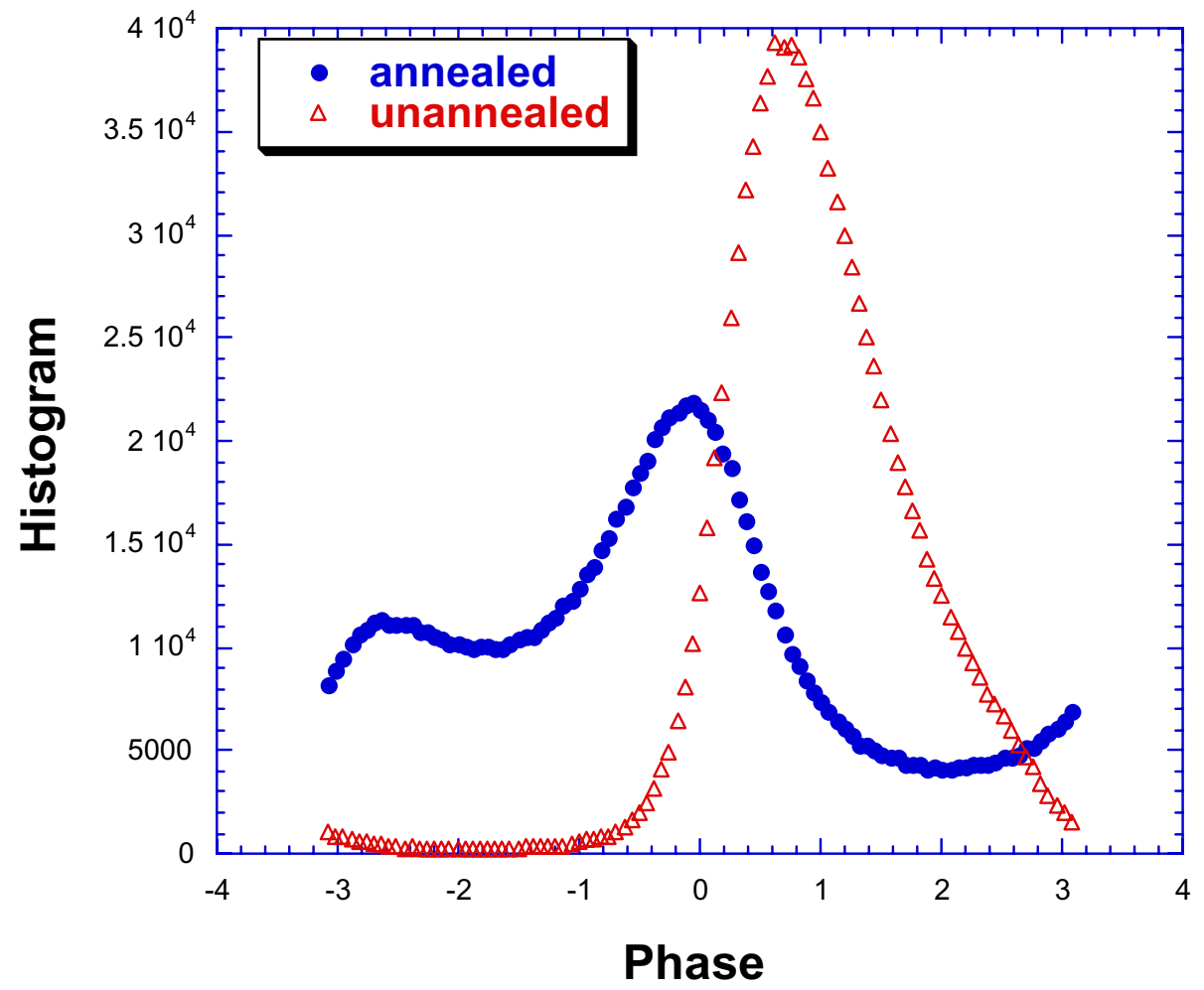

Figure 1. Histograms of the phase for an annealed amorphous diamond-like carbon film and an unannealed one. The electrostatic potential probability distribution may later be retrieved from the histograms. 